\title{
Discussion On Martial Arts Games in Martial Arts Teaching in Ordinary Colleges and Universities
}

\author{
Meili Wang', Yuqin Huan ${ }^{2}$ \\ ${ }^{1}$ Shandong Business and Technology University, Shandong, China \\ ${ }^{2}$ Qingdao Technological University Qindao College, Shandong, China
}

Keywords: Ordinary colleges and universities, Martial arts teaching, Martial arts games.

\begin{abstract}
Martial arts game developed with martial arts. Martial Arts game, as a kind of sports games, can be used in martial arts teaching to greatly improve the teaching results in colleges and universities. Compared with professional martial arts teaching, martial arts teaching in colleges and universities is less professional but focus more on cultural heritage. And martial arts game can enhance interest in teaching and stimulate students' enthusiasm in learning martial arts in colleges and universities. Also, it can reflect martial arts spirit and martial arts culture, which help to build up people's body and cultivate sentiments. Based on this, martial arts game in martial arts teaching in colleges and universities were analyzed and explored in this paper, providing certain reference.
\end{abstract}

\section{Introduction}

Although martial arts is the essence of our country's culture, and occupies a very important position in our country's physical education, it exists in name only in the practice. Aerobics, dance, basketball, soccer and other sports activities are often choose as physical education in ordinary colleges and universities. The phenomenon that students do not like martial arts lesson but martial arts has serious influences on. the development of martial arts teaching in ordinary colleges and universities, which has been an important issue that should be thought about by ordinary colleges and universities. Martial arts game, as the combination of martial arts and games, can greatly stimulate students' interest in martial arts learning, giving martial arts teaching new vitality. Therefore, it is of great significance to discuss and analyze the martial arts game in martial arts teaching.

\section{Introduction of Martial Arts Games}

Martial arts games have a long history of development in our country. Jiao-Di opera, sumo and Cuju are people's favorite martial arts games from the ancient times. Martial arts games come from people's production and living activities. They are not the main body and components of martial arts but develop with the development of martial arts. According to the development of martial arts games and their relationship with martial arts, martial arts games can be defined like this. Martial arts games is a special game activities which can help to enhance physical fitness, cultivate sentiment, and improve martial arts skills, based on martial arts as the basic action and carrying out exercises through the form of game. From the perspective of martial arts teaching, martial arts games are the combination of martial arts and games, which not only contains the information and content of martial arts teaching, but also has obvious characteristics of game entertainment. They can play a good result in martial arts teaching. Also, as educational and recreational activities, they can greatly improve the teaching effect of martial arts.

According to the the equipment used, martial arts games can be divided into the following two categories, namely unarmed martial arts games and armed martial arts games. First of all, unarmed martial arts games, such as individual drill, team drill, free combat, hand-pushing, are those being carried out without equipment. According to the number of participants, unarmed martial arts games 
can also be further divided into single game, double game and collective game. Among them, the double game is an offensive and defensive game mainly for the simulation action of martial arts. Usually, it is an entertainment carried out by kicking, wrestling and other ways in accordance with the rules, relying on the shed squeeze by mining techniques and other techniques. It has a wide range of applications and can be carried out in the process of martial arts teaching at any time. For example, when explaining and demonstrating the actions, teachers can introduce the double game to a better explain the martial arts action and routines to help students understand. The collective game is often used to strengthen the students' memory of martial arts. Students are divided into groups and then participate in the game under the certain topics according to the

rules, which both help to achieve good teaching effect, and allows students enjoy themselves. Secondly, the armed martial arts games refer to those carried out with weapons and equipment. There is a variety of martial arts equipment, and different devices have different technical characteristics and martial arts styles. Samely, in accordance with the number of participants, armed martial arts games can be divided into single equipment game and pair equipment game. . Because the basic requirements of each kind of equipment is different, not only the martial arts routine, but also the use of equipment should be considered and researched in the process of martial arts game creation. Teachers should choose different teaching methods according to the teaching content, so that students can apply martial arts games in the use of martial arts equipment. Then students can know, be familiar with and master using equipment.

According to the structure of the sports curriculum, martial arts games can be divided into preparation activities game, technological martial arts game and relaxation game. Among them, the preparation activities game is carried out in the preparation part, mainly including the entertainment of the collective martial arts game, so as to create a relaxed, entertaining classroom atmosphere. And students can quickly get into the martial arts learning and have friendly relationship with each other. Technological martial arts game is carried out in the basic part of physical education, generally including pair practice, hand attack or martial arts equipment using. Students can better understand the teaching content only when the content of martial arts teaching are closely related to martial arts games. The relaxation game is carried out at the end of the sports course, which is carried out in the form of a warm and happy martial arts game such as the Tai Chi course, the martial arts proverb, and so on. The purpose is to let the students relax, further consolidate the teaching content and enhance the teaching effect. Psychological adjustment is the main purpose of this stage of martial arts game.

\section{The Function of Martial Arts Games in Martial Arts Teaching in Ordinary Colleges and Universities}

Compared with other disciplines, physical education is more lively. In the martial arts class, as an important part of physical education, students should be proactive and lively. But in teaching practice, we found that there is a lot of skipping phenomenon on physical education in many colleges. According to the traditional teaching methods, teachers can not only stimulate students to participate in the martial arts learning, but also depress their characteristic development to a certain extent. The application of martial arts game in martial arts teaching can fully reflect the fitness and recreation of modern physical education teaching, create a relaxed and lively teaching environment by creating different martial arts game teaching situations, and let the students' personality be fully developed.

Through the martial arts games, students can be guided to actively participate in martial arts learning, cultivate the excellent quality and fully grasp and understand the basic movements of martial arts and skills. After reasonable use of martial arts games in the martial arts teaching, students' interests are no longer simply focused on the mastery and understanding of martial arts action, but also on the principle of actions, and then students can closely combine theory and practice.

The development of martial arts games is the cultural communication of martial arts games. Through the martial arts game design, martial arts culture infuses into the games and students can 
experience martial arts culture when participating in martial arts game. The courage, righteousness, benevolence, kindness and zen in martial arts culture can be fully reflected in the game design. For example, in the tai chi teaching process, "Zen" related games can be designed so that students can understand more about "Zen", which helps to inherit the culture.

There is a lot of teaching content and a variety of styles of martial arts. The action and course of martial arts change a lot, so the student's memory will inevitably be chaotic, missing.Martial arts games in the application of martial arts teaching can effectively solve this problem. Simple practice is very boring, which is the main reason that a lot of students do not want to carry out martial arts practice. Martial arts games can break down the actions so that students learn while using. The game can enhance students' interest and enthusiasm, and let students actively participate in the game exercise, continuing strengthening the learning and memory.

\section{The Application of Martial Arts Games in Martial Arts Teaching in Ordinary Colleges and Universities}

College students have strong sense of autonomy. If the martial arts games are inexplicably joined with martial arts teaching project,students may feel confused and not be able to actively participate in them. Therefore, before the start of teaching, teachers should introduce the purpose,meaning and methods of martial arts games to students, so that students have a general understanding of martial arts games, which not only can improve recognition of students on martial arts and help students participate in the design and optimization of games with teachers, making the martial arts games more suitable for them.

Teachers should have a basic understanding and mastery about students' understanding of the game and relative qualities, which can help teachers make reasonable arrangements on teaching objectives and participants and different martial arts games according to the physical characteristics of students. The time, distance and weight should be arranged according to the actual situation rather than pursuing amount of exercise while damaging to the physical and mental health of students.

The teachers should be fully aware of that the continuous application of martial arts games in teaching can help students to accept different things, open thinking. Also, they can constantly innovate martial arts games using their creative ability. In the teaching process teachers should focus on the development of student potential and let students continue to innovate martial arts games, improving the teaching effect, while extending the meaning and meaning of martial arts teaching.

Martial arts culture has a strong influence and communication. Almost every ordinary university has at least one martial arts association, which should be made full use of by teachers to guide the associations to carry out purposeful and planned martial arts cultural activities, including martial arts cultural festival, martial arts cultural exchange and so on. , To encourage students to actively participate in martial arts cultural activities, there should be a clear system, content, and appropriate rewards and punishments. Students should be encouraged to actively participate in martial arts cultural activities, which will not only stimulate students to learn martial arts more seriously, but exercise their interpersonal skills, to achieve the purpose of cultural exchange.

The summary of the experience of martial arts games can play a positive effect in two areas. First of all, through the summary, students can fully understand their own progress. The constant joy of the stage of success can help students to enhance confidence and have more intense interest, which is conducive to the further development of teaching activities. Secondly, it can also provide valuable experience for the future design and development of martial arts games. Teachers can improve the teaching content based on the existing shortcomings, promoting the scientific design of martial arts.

First of all, the evaluation indicators should be added as important curriculum assessments, including the effect, students' favorite degree, and fitness with teaching content to enhance the promotion and practice of martial arts games. Secondly, it is necessary to reflect the evaluation indexes related to the martial arts games in the evaluation of students' learning effect, such as the performance of the classroom and the enthusiasm of the martial arts games, both of which can 
stimulate the enthusiasm of the teachers and students and make martial arts games an important teaching method in ordinary colleges and universities martial arts teaching.

\section{Conclusion}

The application of martial arts games in martial arts teaching can greatly improve the teaching effect and stimulate the enthusiasm and initiative of students to learn martial arts. In the practical application, teachers should rationally and scientifically design the martial arts games based on the characteristics of students and the content of college martial arts teaching, letting it give full play to stimulate students enthusiasm, improve the teaching effectiveness, and constantly improve the teaching effect of college martial arts.

\section{References}

[1] S. B. Sun, C. L. Li. On Martial Arts Culture Education in Martial Arts Teaching in Colleges and Universities [J]. Journal of Educational Institute of Jilin Province (middle), 2015 (1): 116-117.

[2] L. N. Yang. Analysis of the Main Problems and Countermeasures in Martial Arts Teaching in Ordinary Colleges and Universities [J]. Time Education, 2014 (3): 142.

[3] J. Yu. The Application of "Game Teaching Method" in Martial Arts Teaching in Colleges and Universities [D]. Beijing: Beijing Sport University, 2012.

[4] Y. L. Chang. Research on Martial Arts Game in Martial Arts Teaching in Ordinary Colleges and Universities [D]. Fuzhou: Fujian Normal University, 2012.

[5] Y. X.Wu. Analysis of the Present Situation and Countermeasures of Wushu Teaching in Colleges and Universities [J]. Chinese Martial Arts (research), 2013 (8): 63-67.

[6] N. N. Sun, Y. Li. An Analysis of the Current Situation of Martial Arts Teaching in Colleges and Universities in the New Situation [J].Journal of Chifeng University 2014 (14): 98-99. 